\title{
Non-linear Finite-Time Tracking Control of Uncertain Robotic Manipulators Using Time-Varying Disturbance Observer-Based Sliding Mode Method
}

\author{
Hamid Razmjooei ${ }^{1,2}$ (D) $\cdot$ Mohammad Hossein Shafiei $^{1} \cdot$ Gianluca Palli $^{2} \cdot$ Mohammad Mehdi Arefi $^{3}$
}

Received: 24 August 2021 / Accepted: 6 January 2022 / Published online: 11 February 2022

(C) The Author(s) 2022

\begin{abstract}
In this paper, a time-varying chattering-free disturbance observer-based position tracking control law of serial robotic manipulators is presented to track a reference signal in a finite time. The key idea is to employ a positive-increasing function associated with the control/observer objectives to improve the control performance. First, the model of an uncertain robotic manipulator is presented as the case study of the proposed strategy. Then, the time-varying form of the robotic manipulator model is obtained to provide finite-time boundedness using the first-order sliding mode method. Moreover, without any knowledge about the upper bounds of the uncertainties, a reduced-order observer is presented to estimate the uncertainties in a finite time. Subsequently, a disturbance observer-based finite-time position tracking control law is designed. The time-varying gains are provided to converge the position tracking error to a neighborhood of zero in a finite time. Finally, comparative simulations are presented to show the effectiveness of the proposed scheme compared to other existing strategies.
\end{abstract}

Keywords Finite-time tracking control $\cdot$ Nonlinear finite-time observer $\cdot$ Robotic manipulators $\cdot$ Stability analysis $\cdot$ Uncertain time-varying system

\section{Introduction}

The problem of position tracking in uncertain robotic manipulators is a well-known topic over the last years, especially in the presence of external disturbances [1-7]. Many tracking approaches provide asymptotic or exponential convergence

Hamid Razmjooei

h.razmjooei@sutech.ac.ir; hamid.razmjooei@unibo.it

Mohammad Hossein Shafiei

shafiei@sutech.ac.ir

Gianluca Palli

gianluca.palli@unibo.it

Mohammad Mehdi Arefi

arefi@shirazu.ac.ir

1 Department of Electrical and Electronic Engineering, Shiraz University of Technology, Shiraz, Iran

2 Department of Electrical, Energy and Information Engineering, University of Bologna, Bologna, Italy

3 Department of Power and Control Engineering, School of Electrical and Computer Engineering, Shiraz University, Shiraz, Iran (convergence of tracking errors in an infinite time). However, in many robotic's applications, high steady-state precision and finite-time convergence are important due to the precision and safety requirements of the application. Besides, in the presence of unknown terms, finite-time tracking of reference signals precisely is somehow impossible, and instead, the concept of boundedness should be considered [8].

Regarding finite-time boundedness, several strategies have been presented by academic and industrial researchers [9]. However, one of the main drawbacks of these strategies is the need for some information about unknown terms, which is not feasible in many practical situations. In practice, an alternative approach is to measure the outputs of the system and to design an observation algorithm to construct a reliable estimation of the unknown terms. In this regard, disturbance observer-based control schemes have been proposed, in which to decrease the initial value of the control input, the observer is combined with the state feedback law. Some results related to this concept have been presented in [10-14], where the sliding mode observer (SMO)-based control is one of the main methods with widespread applications. Despite the successful deployment of the SMO-based control method, its standard version is affected by some restrictions, leading to the 
potentially destructive chattering phenomenon in their convergence. Besides, an extended state observer (ESO)based super-twisting control method for robotic manipulators with parameter uncertainties and external disturbances has been presented in [10]. Since the sign function is used in super-twisting control approaches, it can lead to the chattering phenomenon. To mitigate this drawback, alternative approaches have been developed, such as the high-order sliding mode observer (HSMO)-based control method and the terminal sliding mode observer (TSMO)-based control method to reduce the chattering with finite-time convergence properties $[5,6,11-14]$. On the other hand, it is desirable to have a fixed convergence time regardless of initial conditions. Fixed-time approach using the concept of bi-limit homogeneity has been introduced in $[15,16]$, where is more powerful than the finite-time approach. However, in addition to structural limitations, the gains are not easily computable. Moreover, finding a proper Lyapunov function to prove stability is not straightforward for high-order systems.

Recently, time-varying observers have been well investigated [17-23]. For instance, some time-varying observers with exponential convergences have been presented in [17-21]. However, their shortcomings are as follows: (1) The estimation errors have not been considered in the control loop. Indeed, the estimation errors should be considered as the uncertainties of the state variables in the stability proof of the closed-loop system. (2) Required prior knowledge about the uncertainties of the system. To overcome these shortcomings, a novel time-varying technique has been presented $[22,23]$. This technique estimates the full state of the system in a finite time and stabilizes it with consideration of the estimation errors. Compared with the existing strategies, its main advantages are the straightforward design and robustness against uncertainties. However, its limitation is the singularity problem when the time tends to infinity. Moreover, it is only applicable to single-input single-output (SISO) canonical systems.

Moreover, a high-performance approach in the field of finitetime observer-based tracking control methods has been presented in [24]. This reference has provided high tracking accuracy, fast response time, low chattering phenomenon, and robustness against uncertainties and external disturbances. Indeed, [24] has presented a third-order sliding mode observer (TOSMO) and has shown its advantages compared to a second-order sliding mode observer (SOSMO). Then, a non-singular fast terminal sliding mode control based on third-order sliding mode observer (NFTSMC-TOSMO) has been presented in [24] and its superiority over conventional non-singular fast terminal sliding mode control (NFTSMC) and non-singular fast terminal sliding mode control based on second-order sliding mode observer (NFTSMC-SOSMO) has been illustrated. Despite several studies and except for this leading strategy, the finite-time observerbased tracking control subject still requires further improvement.

Motivated by the above considerations, this paper presents a new method for finite-time tracking control of robotic manipulators. The key idea is to employ a positive-increasing function associated with the controller and observer objectives to improve the control performance. In this regard, a robust time-varying approach is presented to design a disturbance-observer based finite-time tracking control (DO-FTTC) law, in a simple and straightforward manner. In this paper, the state-space model of the uncertain robotic manipulator system is transformed into a time-varying form, and then, without any knowledge about the upper bounds of the uncertainties, finite-time boundedness of the system is assured using asymptotic stability methods. The time-varying gains of the controller and observer are designed simply to converge the tracking and observation errors to the neighborhood of zero without any chattering and to remain uniformly bounded in a finite time. Finally, to highlight the efficiency of the proposed framework, several comparative simulations are reported between the proposed DO-FTTC method and the superior approach NFTSMC-TOSMO of [24]. Compared with the existing literature, the main contributions of this paper are as follows: (1) Without any knowledge about the upper bound of uncertainties, continuous and chattering-free observer and controller are designed. (2) A time-varying conversion is introduced such that the stabilization and estimation are guaranteed based on finite-time boundedness using the asymptotic stability analyses. (3) Time-varying gains are computed based on straightforward algebraic equations.

The remainder of this paper is organized as follows. In Section 2, a class of robotic manipulators with any number of links is introduced. A state feedback control law is designed in Section 3, to guarantee the position tracking in a finite time. In Section 4, first without, neither any knowledge about the upper bounds of the unknown terms nor its derivative, an observer is designed to estimate the unknown variables in a finite time. Subsequently, the DO-FTTC is designed to converge the tracking errors to the neighborhood of zero without any chattering. In Section 5, simulation results show the effectiveness of the designed DO-FTTC. Finally, conclusions are presented in Section 6.

\section{Preliminaries and Robot Model Description}

Consider a class of serial $p$-link robotic manipulators as follows [8]:

$$
\begin{aligned}
M(\theta) \ddot{\theta}= & \tau^{-} \tau_{d}-C(\theta, \dot{\theta}) \dot{\theta}-F(\theta, \dot{\theta})-G(\theta) \\
& +P\left(t^{-} t_{f}\right) f(t)
\end{aligned}
$$

where $p>0$ is the number of links, $\theta \in R^{p}, \dot{\theta} \in R^{p}$, and $\dot{\theta} \in R^{p}$ denote the position, velocity, and acceleration of robot joints, respectively. Moreover, $M(\theta) \in R^{p} \times p, C(\theta, \dot{\theta}) \in R^{p}$, $F(\theta, \dot{\theta}) \in R^{p}$, and $G(\theta) \in R^{p}$ represent the symmetric and positive definite inertia matrix, the Coriolis and centripetal 
forces, the friction, and the gravitational force, respectively. Besides, $\tau, \tau_{d} \in R^{p}$ represent the control input torque and the unknown bounded external disturbance, respectively. In addition, $f(t) \in R^{p}$ denotes an unknown fault which is assumed as a special case of the disturbance term $\tau_{d}$. In this regard, $P\left(t-t_{f}\right)$ is used in the simulations as the time profile of $f(t)$ where $t_{f}$ is the time of occurrence of the fault. For simplicity, $M(\theta), C(\theta, \dot{\theta}), F(\theta, \dot{\theta}), G(\theta)$, and $f(t)$ hereafter are shown as $M, C, F, G$, and $f$, respectively.

Assumption 1 In this paper, model uncertainties $\Delta M$ and $\Delta G$ are considered in the matrices of the system (1) as $M=M_{0}+\Delta M$ and $G=G_{0}+\Delta G$ where the non-singular matrix $M_{0}$ and the vector $G_{0}$ represent the nominal parts of $M$ and $G$, respectively.

In this paper, $x=[\theta, \theta]^{T}$ is defined as the state vector and the state-space form of the dynamical system (1) is given as:

$$
\begin{aligned}
\dot{\mathrm{x}_{1}} & =x_{2} \\
\dot{x_{2}} & =-M^{-1}\left[C x_{2}+F+G-\tau+\tau_{d}+P\left(t-t_{f}\right) f\right]
\end{aligned}
$$

where $x_{1}=\theta$ and $x_{2}=\dot{\theta}$. Based on Assumption 1 and the matrix inversion lemma [25], one has

$\dot{x_{2}}=-M_{0}^{-1}\left(G_{0}-\tau\right)+\Sigma_{e q}$

where the uncertainties, disturbances, and the unknown fault has been summarized as the following generalized disturbance $\Sigma_{e q}$ :

$$
\begin{aligned}
\Sigma_{e q}= & -\left(M_{0}^{-1} \Delta M M_{0}^{-1}\left(I+\Delta M M_{0}^{-1}\right)^{-1}\right) \\
& \left(C x_{2}+F+G_{0}+\Delta G-\tau+\tau_{d}+P\left(t-t_{f}\right) f\right) \\
+ & M_{0}^{-1}\left(C x_{2}+F+\Delta G+\tau_{d}+P\left(t-t_{f}\right) f\right)
\end{aligned}
$$

The following Definitions and Remark will be used throughout the paper:

Definition 1 [8]: The time-varying system $z=\chi(t, z, \delta)$ is said to be finite-time ultimate bonded (FTUB) in a finite time $T$ with respect to $\delta$ where $\delta^{T} \delta \leq \sigma$, if for some positive-definite matrix $R$ and any positive constants $a, b$, and $\sigma$, where $0 \leq a \leq b$, the condition $z^{T} R z<b$ is satisfied for $t \in[0, T]$ whenever $z_{0}^{T} R z_{0} \leq a$.

Definition 2 [8]: The system $z=\chi(t, z, \delta)$ is said to be finite-time input-to-state stable (FT-ISS) in a finite time $T$ with respect to $\delta$ where $\|\delta\| \leq \sigma$, if the inequality (5) is guaranteed for a class $K L$ function $\alpha$, a class $K$ function $\beta$ and for any $t \geq t_{0}+T$ :

$$
\|z\| \leq \alpha\left(\left\|z_{0}\right\|, \bar{\vartheta}\right)+\beta(\|\delta\|)
$$

Also, $\bar{\vartheta}$ is a time-varying function tending to infinity as $t$ $\rightarrow t_{0}+T$. It is worth noting that, in the absence of the disturbance $\delta$, an FT-ISS system will be finite-time stable.

Remark 1 Consider the change of coordinates $\Sigma_{1} \rightarrow \Sigma_{2}$ in form of $\Sigma_{2}=C \Sigma_{1}$, where $C$ is defined as a positive increasing function diverging to infinity when $t \rightarrow t_{0}+T$. Then, if the variable $\Sigma_{2}$ remains stable (does not tend to infinity), the boundedness of the first coordinate $\Sigma_{1}$ as $t \rightarrow t_{0}+T$ is guaranteed [22].

\section{Finite-Time Tracking Control Law Design}

The main contribution of this paper is to introduce a novel design algorithm so that the output $y$ track the time-varying reference signal $y_{r}$, where $y_{r}$ and its derivatives are known and bounded for all times. First, let us define the tracking error variables $e_{i}$ as follow:

$e_{1}=y-y_{r}=x_{1}-y_{r}$

$$
e_{2}=x_{2}-y_{r}
$$

where $\dot{y}_{r}$ presents the first derivative of the reference signal $y_{r}$. Consequently, the tracking error dynamics are obtained as follow,

$$
\begin{aligned}
& \dot{e_{1}}=e_{2} \\
& \dot{e_{2}}=-M_{0}^{-1}\left(G_{0}-\tau\right)+\Sigma_{e q}-\dot{y_{r}}
\end{aligned}
$$

In this paper, the design process is explained in two main steps. First, by defining a conversion, the tracking error dynamics (7) is transformed into a new time-varying form to provide finite-time boundedness of tracking using the firstorder sliding mode method. Then, the robust finite-time tracking control law is designed. Subsequently, to achieve the main goal of the paper, first without any knowledge about the upper bounds of the uncertainties, a reduced-order observer is presented, and then the DO-FTTC law is designed.

\section{Step 1. Transform into a new coordination}

The procedure begins with the following transformation $e_{i} \rightarrow \bar{e}_{i}$.

$\bar{e}_{i}=\mu_{c} e_{i}$

where $i=1$ and 2 , also $\mu_{c}\left(t, T_{c}\right)=\frac{1+e^{\frac{-t}{T_{c}}}}{2 e^{\frac{t}{T_{c}}}}$ is defined as a time-varying function (for simplicity shown as $\mu_{c}$ ). $T_{c}$ is a positive design parameter and is selected later for finite-time convergence. Let us calculate the derivative of $\bar{e}_{i}$ along with (7) as

$\dot{\bar{e}_{i}}=\mu_{c} \dot{e}_{i}+\dot{\mu}_{c} e_{i}$ 
where $\dot{\mu}_{c}=\frac{1-\mu_{c}^{-1}}{2 T_{c}}$. After lengthy, but straightforward calculations, the following results are obtained,

$\dot{\bar{e}}_{1}=\frac{\dot{\mu}_{c}}{\mu_{c}} \bar{e}_{1}+\bar{e}_{2}$

and

$\dot{\bar{e}}_{2}=\frac{\dot{\mu}_{c}}{\mu_{c}} \bar{e}_{2}+\mu_{c}\left(-M_{0}^{-1}\left(G_{0}-\tau\right)+\Sigma_{e q}-\dot{y}_{r}\right)$

Based on Remark 1, if the stability of $\bar{e}_{i}$ is achieved, the finite-time stability of $e_{i}$ dynamics will be guaranteed. In fact, the condition that the closed-loop system (10), (11) does not have unstable behavior is sufficient to conclude that the dynamic system (7) is finite-time ultimate bounded.

\section{Step 2. Designing the control law $\tau$}

In this section, the aim is to design a control law to stabilize the transformed error dynamics (10), (11). First, it is assumed that $\Sigma_{e q}=0$, and the finite-time tracking control (FTTC) law for the nominal robot system is designed.

Now, based on the sliding mode control (SMC) method, the asymptotic stability of the system (10), (11) is investigated. Generally, there are two phases to design an SMC law (reaching and sliding phases) [2]. The first phase is to design a proper switching surface function $s$ to drive the resulting sliding motion to the origin. The second phase is to design an appropriate SMC law to converge the state trajectories of the system onto the predefined sliding surface and maintain them there for all subsequent time [2]. In this regard, based on $\bar{e}_{i}$ 's dynamics, consider the sliding surface candidate (12):

$s=\bar{e}_{2}+a \bar{e}_{1}$

With the following FTTC law:

$\tau=G_{0}-\mu_{c}{ }^{-1} M_{0}\left(\left(\frac{\dot{\mu_{c}}}{\mu_{c}}+\lambda_{1}\right) s+a \bar{e}_{2}-\mu_{c} \dot{y_{r}}\right)$

where $a$ and $\lambda_{1}$ are positive constants and will be designed later, the closed-loop nominal form of (10), (11) is written as

$\dot{\bar{e}}_{1}=\frac{\mu_{c}}{\mu_{c}} \bar{e}_{1}+\bar{e}_{2}$

$\dot{\bar{e}_{2}}=-\left(\frac{\dot{\mu}_{c}}{\mu_{c}}+\lambda_{1}\right) a \bar{e}_{1}-\left(\lambda_{1}+a\right) \bar{e}_{2}$

Now, the main result of this section is given as the following Theorem:

Theorem 1 Consider the nominal form of the robotic manipulators (2). If $\tau$ is designed as the FTTC law (13), there exist suitable parameters $a>\underline{\mu_{c}} \mu_{c}$ and $\lambda_{1}$ such that the variable $y$ and $\dot{y}$ will track the time-varying reference signals $y_{r}$ and $\dot{y}_{r}$, respectively, in a finite time.

Proof. Consider the candidate Lyapunov function as $V=\frac{1}{2} s^{2}$. Then, the time derivative of this Lyapunov function along the closed-loop system (14) is

$\dot{V}=s \dot{s}=-\lambda_{1} s^{2}$

According to the definition of Lyapunov function $V$, (15) can be rewritten as

$\dot{V}=-2 \lambda_{1} V$

where the trajectory of the closed-loop system (14) reaches the sliding surface $s=0$, and once on the surface $s$, it can not leave it. Therefore, the reaching phase is completed.

Then, using (12) and (14), the motion on the surface $s$, is governed by the following reduced-order model:

$\dot{\bar{e}}_{1}=\left(\frac{\dot{\mu_{c}}}{\mu_{c}}-a\right) \bar{e}_{1}$

where choosing the positive constant $a$ as $a>\underline{\mu}_{c} \mu_{c}$, leads to the stability of the reduced-order model (17), such that the state variable $\bar{e}_{1}$ and subsequently $\bar{e}_{2}$ tends to zero as $t$ tends to infinity along $s=0$. Subsequently, the sliding phase motion is ensured and the stability of the sliding surface $s$ is guaranteed [2]. Therefore, based on the stability of the reduced-order model (17) and the structure of $s$, it can be concluded that $\bar{e}_{i}$ 's remain uniformly bounded. Since the error $\bar{e}_{i}$ converges into a small neighborhood around zero and according to the transformation (8), the error $e_{i}$ converges into a small neighborhood around zero in a finite time. To complete the proof, now consider the following compact form of the closed-loop system (14),

$\dot{\bar{e}}=\Lambda_{c} \bar{e}$

where $\Lambda_{c}$ is defined as

$\Lambda_{c}=\left[\frac{\dot{\mu}_{c}}{\mu_{c}} 1-\left(\frac{\dot{\mu}_{c}}{\mu_{c}}+\lambda_{1}\right) a-\left(\lambda_{1}+a\right)\right]$

the constants $a>\frac{\mu_{c}}{\mu_{c}}$ and $\lambda_{1}$ are chosen to make the matrix $\Lambda_{c}$ Hurwitz for all times. Thus, there exists a positive constant $\varepsilon_{1}>1$, such that

$\|\bar{e}\| \leq \varepsilon_{1}\left\|e^{\Lambda_{c} t}\right\|\|\bar{e}(0)\|$

Therefore, the mentioned sufficient condition is achieved and based on Remark 1, since the stability of $\bar{e}_{i}$ has been achieved, the finite-time stability of $e_{i}$ dynamics is guaranteed. Now, based on the transformations (8) one has 


$$
\|e\| \leq v_{c}\|\bar{e}\|
$$

where $v_{c}=\mu_{c}^{-1}$. Moreover, based on the transformations (8) since

$\|\bar{e}(0)\| \leq\|e(0)\|$

by straightforward calculations, the following zone-convergence will be achieved.

$\|e\| \leq v_{c} \varepsilon_{1} e^{\Lambda_{c} t}\|e(0)\|$

where $v_{c}$ was already defined as a positive un-incremental function converging to an arbitrarily small zone around zero as $t \rightarrow t_{0}+T$. Therefore, the FTUB stability of tracking errors $e$ in (7) within a bounded time interval is guaranteed.

Since $\mu_{c}$ and consequently $\frac{\mu_{c}}{\mu_{c}}$ are time-varying, satisfying the conditions on $a$ and $\lambda_{1}$ may be difficult. In this regard, Corollary 1 is proposed to derive a sufficient condition to guarantee the conditions.

Corollary 1 Based on $\mu_{c}$ and its time derivative, one has $\frac{\mu_{c}}{\mu_{c}}=\frac{1}{2 T_{c}}\left(1-\mu_{c}^{-1}\right)$. Since $1 \leq \mu_{c}<\infty, 0<\frac{\mu_{c}}{\mu_{c}} \leq \frac{1}{2 T_{c}}$. Now, the following results are given:

(1) The condition $a>\frac{\mu_{c}}{\mu_{c}}$ in the stability of the sliding surface $s$ can be replaced with $a>\frac{1}{2 T_{c}}$.

(2) Since the time-varying matrix $\Lambda_{c}$ in (19) is continuous and bounded as $\lim _{t \rightarrow \infty} \Lambda_{c}=\bar{\Lambda}_{c}$, the stability of the closed-loop system (14) will be guaranteed if all the Eigenvalues of $\bar{\Lambda}_{c}$ are located on the open left-hand half of the complex plane [22], where $\bar{\Lambda}_{c}$ is calculated as:

$\bar{\Lambda}_{c}=\left[\begin{array}{cc}\frac{1}{2 T_{c}} & 1 \\ -\left(\frac{1}{2 T_{c}}+\lambda_{1}\right) a & -\left(\lambda_{1}+a\right)\end{array}\right]$

Now, the FTTC law (13) has been designed for the nominal form of the robotic manipulator (2). The next task is to design a robust control law for the uncertain robotic manipulator such that the variable $y$ and $y$ track the time-varying reference signals $y_{r}$ and $\dot{y_{r}}$, respectively, in the presence of uncertainties.

\section{Disturbance Observer-Based Finite-Time Tracking Control Design}

In this Section, a robust continuous and chattering-free control law is designed to guarantee the FTUB of the tracking error dynamics (7). First, based on [2] assume that $\tau=\tau_{\text {nominal }}+$ $\tau_{\text {Robust }}$ On the other hand, the generalized disturbance $\Sigma_{e q}$ in (4) is a time-varying function of the state variables and the input vector satisfying the inequality $\left\|\Sigma_{e q}\right\| \leq \rho(t, x)+$ $k \tau_{\text {Robust }}$ [2]. In this regard, $\tau_{\text {nominal }}$ is the nominal control law to guarantee finite-time stability in the absence of unknown terms. The function $\rho(t, x)$ and the parameter $k \in[0$, 1) are the only information we need to know about $\Sigma_{e q}$ (this limitation will be relaxed later). The nonnegative continuous function $\rho(t, x)$ is not required to be small, but it should be just known. Subsequently, based on the Lyapunov redesign technique [2], the FTTC law (13) can be updated as the following robust FTTC law:

$\tau=G_{0}-\mu_{c}^{-1} M_{0}\left(\left(\frac{\dot{\mu_{c}}}{\mu_{c}}+\lambda_{1}\right) s+a \bar{e}_{2}-\mu_{c} \dot{y_{r}}\right)+\tau^{\nu}$

where $\tau$ is added to cancel out the destabilizing effect of the generalized disturbance $\Sigma_{e q}[2,3]$. Although using the robust FTTC law (25), the tracking errors converge to an arbitrarily small zone around zero in a finite time, the information on the unknown terms is assumed to be unknown and not available online. This limitation will be relaxed in this section. In this regard, the Lipschitz continuous condition $\dot{\Sigma}_{\text {eq }} \leq \sigma$ with a known Lipschitz constant $\sigma$ is considered [4, 8]. This condition may often exist in many real-life systems [2]. To design a disturbance observer-based control law, first, the following observer, which is a direct consequence of Theorem 2 of [8], is presented where the convergence of the estimated generalized disturbance $\Sigma_{e q}$ to the actual value is guaranteed in a finite time [8].

$$
\begin{aligned}
\dot{\widehat{x}}_{1} & =\widehat{x}_{2}+k_{1}(t)\left[x_{1}-\widehat{x}_{1}\right] \\
\dot{\hat{x}}_{2} & =\widehat{x}_{3}-M_{0}^{-1}\left(G_{0}-\tau\right)+k_{2}(t)\left[x_{1}-\widehat{x}_{1}\right] \dot{\widehat{x}}_{3} \\
& =k_{3}(t)\left[x_{1}-\widehat{x}_{1}\right]
\end{aligned}
$$

where $\widehat{x}_{i} \in R^{p}$ (for $i=1,2$, and 3 ) are estimations of the state variables, and the time-varying scalar gains $\left\{k_{i}(t)\right\}_{i=1}^{3}$ are functions of the real-time $t$, shown as $k_{i}$ hereafter for simplicity. The gains of the time-varying observer are calculated as follows to achieve convergence in a finite time [8]:

$k_{i}=L_{i}+\bar{k}_{i, 1} \frac{2+i}{2 T_{o}} \mu_{o}^{i-1}\left(1-\mu_{o}^{-1}\right)-\bar{k}_{i+1,1} \mu_{o}^{i}-\sum_{j=1}^{i-1} \bar{k}_{i, j} \mu_{o}^{i-j} k_{j}(27)$

where $i=1$ and 2 also,

$k_{3}=L_{3}+\bar{k}_{3,1} \frac{5}{2 T_{o}} \mu_{o}^{2}\left(1-\mu_{o}^{-1}\right)-\sum_{j=1}^{2} \bar{k}_{3, j} \mu_{o}^{3-j} k_{j}$

also $\left\{\bar{k}_{i, j}\right\}$ are given as follows: 


$$
\begin{aligned}
\bar{k}_{i, j-1}= & \bar{k}_{i, j} \frac{3}{2 T_{o}} \mu_{o}^{-1}\left(1+\mu_{o}^{-1}\right)-\bar{k}_{i, j} \frac{i-j}{2 T_{o}} \mu_{o}^{-1}\left(1-\mu_{o}^{-1}\right) \\
& +k_{i+1, j}
\end{aligned}
$$

and

$\bar{k}_{3, j-1}=\bar{k}_{3, j} \frac{\mu_{o}^{-1}}{2 T_{o}}\left(6 \mu_{o}^{-1}+j\left(1-\mu_{o}^{-1}\right)\right)$

where $\left\{\bar{k}_{i, i}\right\}=1$ and for $i<j$ one has $\left\{\bar{k}_{i, j}\right\}=0$. Also, the scalar coefficients $\left\{L_{i}\right\}$ (for $i=1,2$, and 3 ) are selected to make the matrix $\Lambda_{o}=\left[\begin{array}{cc}-L_{1} & \boldsymbol{I}_{\mathbf{2}} \\ -L_{2} & \\ -L_{3} & 0\end{array}\right]$ Hurwitz where $\boldsymbol{I}_{\mathbf{2}}$ is the 2 $\times 2$ identity matrix [8]. Also, are time-varying functions $\mu_{o}\left(t, T_{o}\right), \mu\left(t, T_{o}\right)$, and $v_{o}\left(t, T_{o}\right)$ (for simplicity shown as $\mu_{o}, \mu$ and $v_{o}$, respectively) are defined as $\mu_{o}=\frac{1+e^{\frac{-t}{T_{o}}}}{e^{\frac{-t}{T_{o}}}}, \mu=$ $\mu_{o}{ }^{3}$, and $v_{o}=\mu_{o}^{-1}$. Also $T_{o}$ is a positive integer, and should be selected to improve the convergence rate. As a sufficient condition, if the design process is done in a way that the eigenvalues of $\Lambda_{o}$ will be placed on the left side of $\frac{-3}{T_{o}}$, the estimation condition is guaranteed [8]. The proof is a direct consequence of Theorem 2 in [8], hence, it is omitted.

\subsection{Disturbance Observer-Based Finite-Time Tracking Control Design}

In this Section, the continuous and chattering-free FTTC law (13) is updated as follows via the disturbance observer-based control approach:

$\tau=G_{0}-\mu_{c}^{-1} M_{0}\left(\left(\frac{\dot{\mu}_{c}}{\mu_{c}}+\lambda_{1}\right) s+a \bar{e}_{2}-\mu_{c} \dot{y_{r}}+\mu_{c} \widehat{\Sigma}_{e q}\right)$

where $\widehat{\Sigma}_{e q}=\widehat{x}_{3}$ is generated by the observer (26). After substituting the DO-FTTC law (31), the closed-loop form of (10), (11) is written as,

$$
\begin{aligned}
& \dot{\bar{e}}_{1}=\frac{\mu_{c}}{\mu_{c}} \bar{e}_{1}+\bar{e}_{2} \\
& \dot{\bar{e}}_{2}=-\left(\frac{\dot{\mu}_{c}}{\mu_{c}}+\lambda_{1}\right) a \bar{e}_{1}-\left(\lambda_{1}+a\right) \bar{e}_{2}+\mu_{c} e_{3}^{\prime}
\end{aligned}
$$

where $e_{3}^{\prime}=x_{3}-\widehat{x}_{3}$. The main result of this section is given in the following Theorem:

Theorem 2 Consider the robotic manipulator (2). If $\tau$ is designed as the DO-FTTC law (31), where $\widehat{\Sigma}_{e q}$ is generated by the observer (26), then the variable $y$ and $y$ track the time- varying reference signals $y_{r}$ and $\dot{y_{r}}$, respectively, in a finite time.

Proof. According to the designed observer (26), there exists a finite time (for instance $T_{o}$ ), such that the estimation error variables approach a bound around the origin and eventually converge to zero, such that $\widehat{x}_{i}=x_{i}$ for $t \gg T_{o}$ [9]. As a result, the closed-loop system (32) coincides with (14) for all $t \gg T_{o}$. Besides, according to [22], if the system's states under the proposed DO-FTTC (31) does not escape during $t \gg T_{o}$, based on Theorem 1 there exists a finite time (for instance $T_{c}$ ) that the robust FTUB stability of tracking errors $e$ in (7) will be guaranteed. Therefore, it is sufficient to show that the closed-loop system (32) under DO-FTTC (31) does not escape in a finite time. In the following, consider the derivative of the time-varying sliding surface (12) along with the trajectory of the closed-loop system (32) so that after straightforward calculations, it can be shown,

$\dot{s}=-\lambda_{1} s+\mu_{c} e_{3}^{\prime}$

Since the variable $\mu_{c}$ is a positive function, (33) has a bounded limit. For the convenience of the proof, depending on the variable $s$ two different cases may occur:

Case $1(s \geq 0)$ : In this case, since $\lambda_{1}$ and $s$ are positive, $s$ in (33) satisfies the following inequality:

$\dot{s} \leq \mu_{c} e_{3}^{\prime}$

Case $2(s<0)$ : In this case, since $\lambda_{1}$ is positive and $s$ is negative, thus, $-\lambda_{1} s>0$, and $s$ in (33) satisfies the following inequality:

$\dot{s}>\mu_{c} e_{3}^{\prime}$

On the other hand, since the FTUB of whole estimation error variables was guaranteed in [8], there exists an upper bound of $e_{3}^{\prime}$ in (34) and (35) (namely $\Pi=\sup \left\{e_{3}^{\prime}\right\}$ ). It is not required that this upper bound be small but only to be known and it can be calculated using the known Lipschitz constant $\sigma$. Therefore, for $s \geq 0$ and $s<0, s$ is bounded as $\dot{s} \leq \mu_{c} \Pi$ and $\dot{s}>\mu_{c} \Pi$, respectively. Therefore, the system state variables as well as $s$, cannot escape in any finite time interval. Finally, since the system's states under the proposed DO-FTTC (31) does not escape during $t \gg T_{o}$, the robust FTUB of the corresponding tracking error system (7) is guaranteed.

Remark 2 Although based on the structure of $v_{o}=\mu_{o}^{-1}$, the asymptotical stability of the estimation error variables is guaranteed, according to the analysis of the designed observer (26) 
in [8], it was only evident that the estimation errors tend to an arbitrarily small zone around zero. Thus, this ultimate boundedness effect in the estimation error should be explicitly considered in the proof of Theorem 2; where it may act as a small uncertainty in the values of the state variables. In this regard, the continuous and chattering-free DO-FTTC law (31) is updated as,

$\tau=G_{0}-\mu_{c}^{-1} M_{0}\left(\left(\frac{\dot{\mu_{c}}}{\mu_{c}}+\lambda_{1}\right) s+a \bar{e}_{2}-\mu_{c} \dot{y}_{r}+\mu_{c} \widehat{\Sigma}_{e q}+\mu_{c} \Theta\right)$ where the auxiliary term $\Theta$ should be designed to suppress the effect of the estimation error so that the closed-loop system remains ultimately bounded as well. After substituting the modified DO-FTTC law (36) into $\dot{s}=\dot{\bar{e}}_{2}+a \dot{e}_{1}$, it is obtained as

$$
\begin{aligned}
\dot{s}= & \frac{\dot{\mu}_{c}}{\mu_{c}} \bar{e}_{2} \\
& +\mu_{c}\left(-M_{0}^{-1}\left(\mu_{c}^{-1} M_{0}\left(\left(\frac{\dot{\mu_{c}}}{\mu_{c}}+\lambda_{1}\right) s+a \bar{e}_{2}-\mu_{c} \dot{y}_{r}+\mu_{c} \widehat{\Sigma}_{e q}+\mu_{c} \Theta\right)\right)+\Sigma_{e q}-\dot{y}_{r}\right) \\
& +a\left(\frac{\dot{\mu}_{c}}{\mu_{c}} \bar{e}_{1}+\bar{e}_{2}\right)
\end{aligned}
$$

with straightforward calculations,

$\dot{s}=-\lambda_{1} s+\mu_{c} e_{3}^{\prime}-\mu_{c} \Theta$

Since there has existed a known upper bound $\Pi$, under the condition $\Theta \geq \sup \left\{e_{3}^{\prime}\right\}$, (38) is simplified as $\dot{s} \leq-\lambda_{1} s$ where based on the Lyapunov function $V$, it can be rewritten as $\dot{V} \leq-2 \lambda_{1} V^{2}$. Therefore, the FTUB stability of tracking errors (7) under the modified DO-FTTC law (36) is guaranteed.

\subsection{Implementation Issues}

One concern of the presented scheme is the definition of the time-varying transformations $\mu_{c}$ and $\mu_{o}$, in which their values can tend to infinity when $t \rightarrow \infty$. To overcome this concern, their definition can be modified as,

$$
\begin{aligned}
& \mu_{c}= \begin{cases}\frac{1+e^{\frac{-t}{T_{c}}}}{2 e^{\frac{-t}{T_{c}}}} & t \leq \delta_{C t} \\
\mu_{C-M a x} & t>\delta_{C t}\end{cases} \\
& \mu_{o}= \begin{cases}\frac{1+e^{-\frac{t}{T_{o}}}}{e^{\frac{-t}{T_{o}}}} & t \leq \delta_{O t} \\
\mu_{O-\operatorname{Max}} & t>\delta_{O t}\end{cases}
\end{aligned}
$$

where for positive real constants $\delta_{C t}$ and $\delta_{O t}$, the constants $\mu_{C}-$ Max and $\mu_{O}-$ Max $_{\text {are nominated as }} 1+e^{\frac{-\delta_{C t}}{T_{c}}} / 2 e^{\frac{-\delta_{C t}}{T_{c}}}$ and $1+e^{\frac{-\delta O_{O}}{T_{c}}} / 2 e^{\frac{-\delta O_{t}}{T_{c}}}$, respectively. The constants $\delta_{C t}$ and $\delta_{O t}$, should be designed based on trade-offs between the tracking errors, observation errors, and the convergence time. To adapt the stability proofs to these new definitions, we consider two intervals $t \leq \max \left\{\delta_{C t}, \delta_{O t}\right\}$ and $t>\min \left\{\delta_{C t}\right.$, $\left.\delta_{O t}\right\}$. For the former, the previous proofs are valid. Also, since we have already proved that the observed states remain within the ball in a finite time, and remain bounded at all times, this statement will remain valid for the second time interval.

In terms of potential challenges about the implementation of the proposed scheme on hardware the following remark is presented:

Remark 3 In terms of implementation, the proposed scheme is straightforward to design and realize. Its construction relies only on choosing the constants $L_{1}, L_{2}$, and $L_{3}$ to make the matrix $\Lambda_{o}$ Hurwitz, and then choosing the constants $T_{c}$ and $T_{o}$, independently. On the other hand, the time-varying observer (26), its gains, and the DO-FTTC law (36) have been computed based on some ordinary algebraic equations, which can be implemented on usual hardwares and it will not need powerful processors. Moreover, the proposed DO-FTTC law (36) is designed using only $\theta$ and $\dot{\theta}$, where $\theta$ have often been measured online in robotic manipulators. However, in terms of the sensor issues, measuring 
or computing the time derivative of $\theta$ (i.e. $\dot{\theta}$ ) appear as a potential challenge of the proposed scheme. To mitigate these challenges and to reduce the number of sensors, an alternative approach is measuring the output $\theta$ with usual sensors and using the observer (26) to construct a reliable estimation of $\dot{\theta}$.

\section{Simulation Results}

In this Section, numerical simulations are presented to clarify the effectiveness of the proposed scheme. First, comparative simulations are reported between the proposed observer and the TOSMO in [24], which has had superior performance to estimate the uncertainties for robotic manipulators. Then, to highlight the efficiency of the proposed scheme to track reference signals in a finite time, comparative simulations are presented between the proposed DO-FTTC and the privileged approach NFTSMCTOSMO. The parameters for the observer are the same as the 2-link robotic manipulator parameters, which the explicit values were provided as follows $[8,9]$ :

$$
\begin{gathered}
M=\left[\begin{array}{cc}
32 \pm \varepsilon \cos \left(\theta_{2}\right) & 0 \\
0 & 13.2 \pm \varepsilon
\end{array}\right], \quad F=\left[\begin{array}{c}
0.8 \dot{\theta_{1}}+1.2 \cos \left(3 \theta_{1}\right) \\
1.3 \dot{\theta}_{2}+0.5 \sin \left(5 \theta_{2}\right)
\end{array}\right] \\
G=g\left[\begin{array}{c} 
\pm \varepsilon \\
13.2 \theta_{2} \pm \varepsilon
\end{array}\right], \tau_{d}=\left[\begin{array}{c}
1.2 \sin \left(0.95 \dot{\theta}_{1}\right) \\
0.9 \cos \left(1.7 \dot{\theta}_{2}\right)
\end{array}\right], f=\left[\begin{array}{c}
2 \cos (0.8 t) \\
3 \sin (t)
\end{array}\right] \\
P(t-3)=\left\{\begin{array}{cc}
0 & t \leq 3 \\
1-e^{-(t-3)} & t \geq 3
\end{array}\right.
\end{gathered}
$$

where $g=9.806 \mathrm{~m} / \mathrm{s}^{2}$ is the acceleration of gravity. Also, based on Assumption 1, $M_{0}=\left[\begin{array}{cc}32 & 0 \\ 0 & 13.2\end{array}\right]$ and $G_{0}=g$ $\left[\begin{array}{c}0 \\ 13.2 \theta_{2}\end{array}\right]$ are the nominal parts of matrix $M$ and vector $G$, respectively. Besides, $\Delta M= \pm \varepsilon\left[\cos \left(\theta_{2}\right)\right.$ 001] and $\Delta G= \pm \varepsilon g\left[\begin{array}{l}1 \\ 1\end{array}\right]$ are the model uncertainties where $\varepsilon$ $\in[0,1]$ is a random variable [9]. The independence property of the proposed method from the upper bounds of the uncertainties has been theoretically presented in sections 3 and 4, where the compensator term $\Theta$ is designed to suppress the effect of the estimation error, where $\Theta \geq \sup \left\{e_{3}^{\prime}\right\}$. In this regard, Based on the Lipschitz continuous condition $\dot{\Sigma}_{e q} \leq \sigma, \sigma$ is the only information we need to know about $\Sigma_{e q}$, where this is a nonnegative Lipschitz constant, and it is not required to be small bounded, but should be just known. The simulations are divided into two parts and all simulation results are obtained with $T_{o}=T_{c}=10$.
Regardless of the variable $T_{o}$, increasing the value of $T_{c}$ reduces the control effort considerably, however, it increases the convergence time significantly and vice versa. The first part (Figs. 1 and 2) shows the efficiency of the proposed observer (26) compared to the TOSMO approach in [24]. The second part (Figs. 3, 4 and 5) shows the closedloop efficiency of the proposed DO-FTTC law (36) compared to the NFTSMC-TOSMO approach. In this regard, the performance analysis of the proposed observer (26) is first shown in Figs. 1 and 2 for the open-loop system $(\tau=$ $0)$. The following two considerations can be drawn: (1) Finite-time convergence is achieved. Figure 1 shows the time evolutions of state variables and their estimations, in which the proposed scheme has obtained an acceptable convergence time compared to TOSMO. (2) The proposed observer results in the proper estimation of the state variables, as well as the uncertainties, whose observation errors converge to a small neighborhood of zero. Figure 2 shows that the proposed scheme achieves comparable estimation performance with respect to TOSMO, even in the presence of unknown terms. The performance of the studied approaches is summarized in Table 1. Performance index $J_{e^{\prime}}$ is defined based on the RMS of the observation error matrix $e^{\prime}=\left[\begin{array}{ll}e_{1}^{\prime} & e_{2}^{\prime}\end{array}\right]=\left[\left\|e_{11}^{\prime}\right\|\left\|e_{12}^{\prime}\right\|\left\|e_{21}^{\prime}\right\|\left\|e_{22}^{\prime}\right\|\left\|e_{31}^{\prime}\right\|\right.$ $\left.\left\|e_{32}^{\prime}\right\|\right]$, as $J_{e^{\prime}}=\left[\left\|\int_{\theta_{1}^{\prime}}^{t_{s}^{\prime}} e_{1}^{\prime} d t\right\|,\left\|\int_{\theta_{2}^{\prime}}^{s_{s}^{\prime}} e_{2}^{\prime} d t\right\|\right]^{T}$, where $t_{s}=$ $20 \mathrm{sec}$ is the simulation time. It is worth noting that, to produce a fair analysis, TOSMO's parameters have been taken directly from [24] with its highest efficiency.

Table 1 confirms that the proposed scheme can compete with the TOSMO approach as a leading strategy, where the proposed observer results in the appropriate estimation of the state variables as well as the uncertainties.

Now the performance of the proposed DO-FTTC law (36) to finite-time tracking of the reference signals $y_{11 r}=\cos (t)$ and $y_{12 r}=-\cos (t)$, by position variables $y=x_{11}$ and $y=x_{12}$ is evaluated. Figures 3 and 4 show that the proposed DOFTTC law (36) ensures a better tracking performance compared with the NFTSMC-TOSMO in [24], which its high tracking precision had been analyzed in [24] compared with conventional NFTSMC and NFTSMC-SOSMO methods.

By defining performance indexes $J_{e}=\left\|\int_{\theta}^{t} e d t\right\|$ and $J_{\tau}=\left\|\int_{\sigma^{T}}^{s} \tau d t\right\|$ based on the infinity norm of the input and tracking error vectors $\tau=\left[\left\|\tau_{\mathbf{1}}\right\| \quad\left\|\tau_{\mathbf{2}}\right\|\right]$ and $e=\left[\left\|e_{11}\right\|\right.$ $\left.\left\|e_{12}\right\|\right]$ respectively, the performance of studied approaches is evaluated in Table 2 .

According to Table 2 and Figs. 3, 4 and 5, the superior performance of the proposed scheme is verified in terms of 

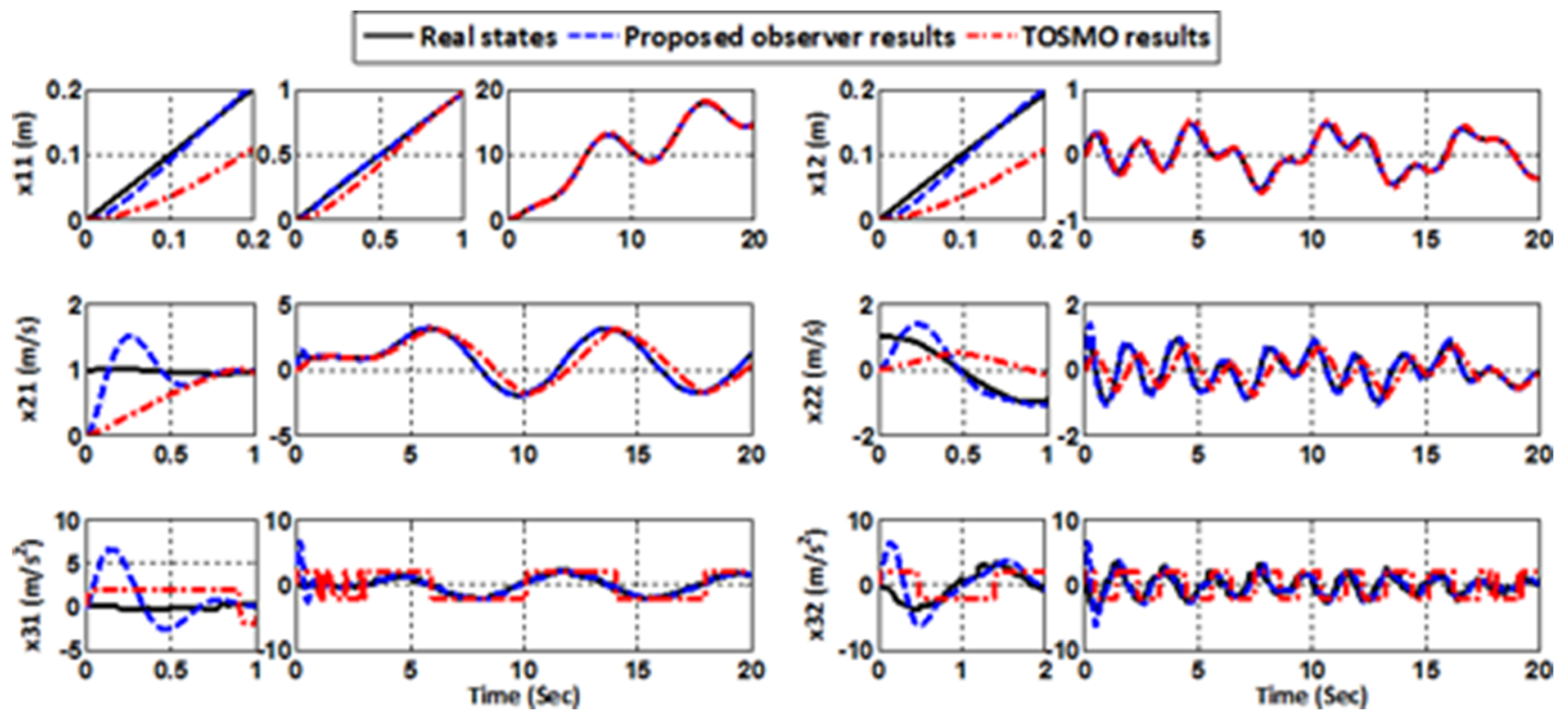

Fig. 1 Real states and estimation results of the open-loop system

convergence time, destructive chattering phenomenon, and control efforts. The finite-time performances have been guaranteed without any singularity problem and it has a smaller convergence time to estimate the uncertainties (less than $1 \mathrm{sec}$ ) and to track the reference signals (less than $2 \mathrm{sec}$ ) compared to leading approaches [24]. Besides, the DO-FTTC law (36) was designed as a continuous (chattering-free) state feedback control law that without any knowledge about the upper bounds of the uncertainties, the variable $y$ and $y$ track the time-varying reference signals $y_{r}$ and $\dot{y}_{r}$, respectively, in a finite time. Finally, the DO-FTTC law (36) has good tracking performance as a finite-time observer-based control approach; where without any knowledge about the upper bounds of the uncertainties, it has almost $30 \%$ less control effort. Therefore,
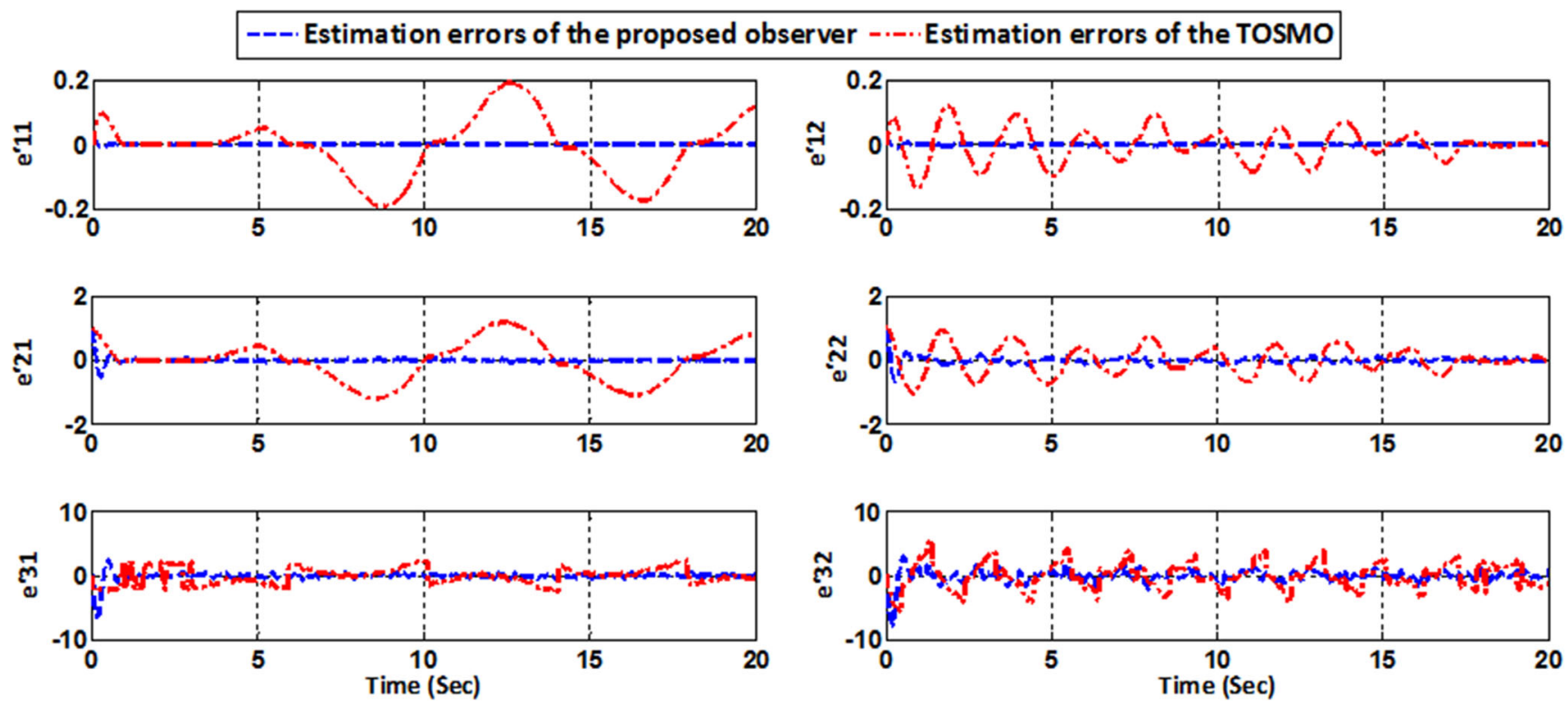

Fig. 2 State estimation errors of the open-loop system 

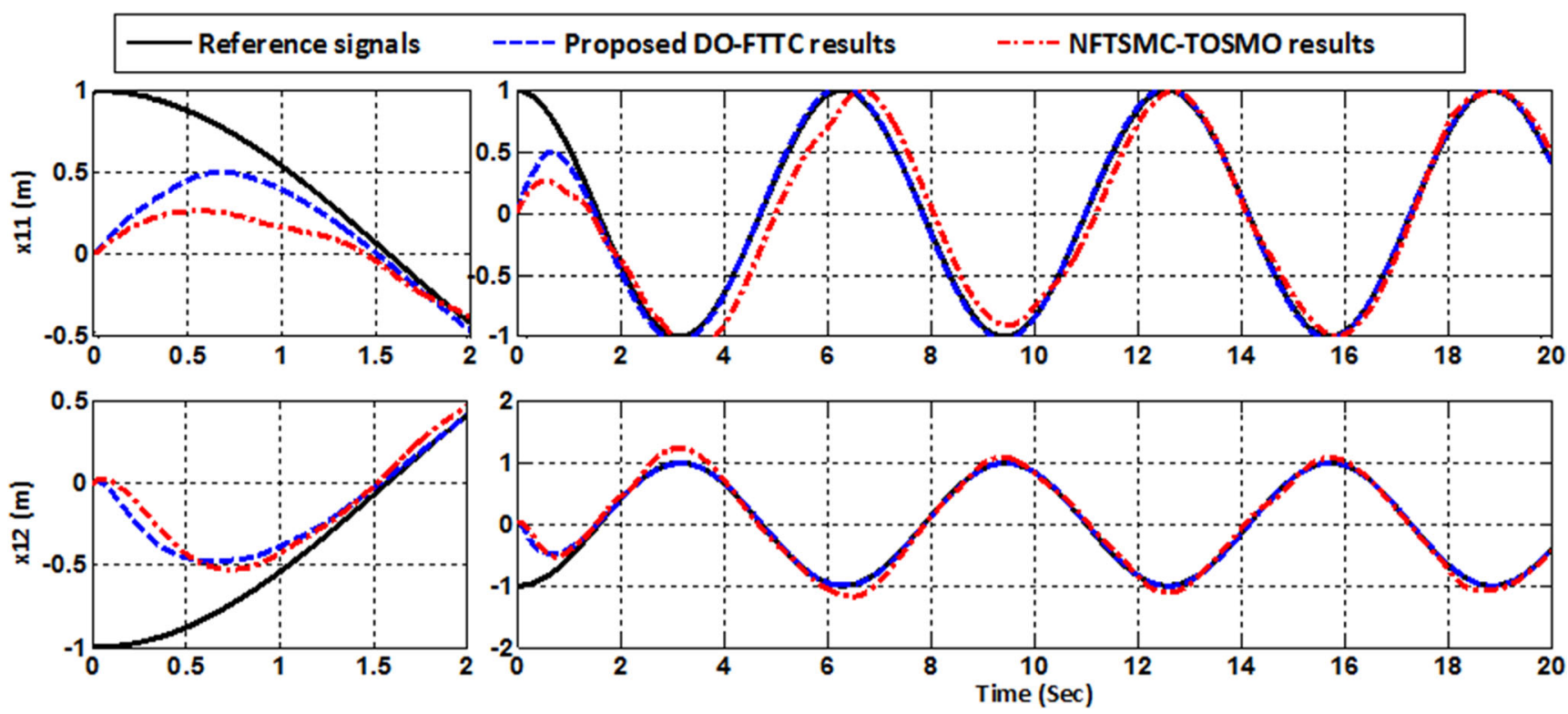

Fig. 3 Reference signals and position tracking results of the closed-loop system

according to the following remark, the proposed scheme can compete with the leading strategy NFTSMC-TOSM, which makes it a qualified alternative approach in the observer and controller design.

Remark 4 One of the main drawbacks of the previous disturbance observers is that the upper bound of the unknown terms is often assumed to be known, which is not feasible in practical situations. On the other hand, the sliding mode-based approaches are frequently affected by the chattering phenomenon. To overcome this problem, a time-varying approach has been presented. Also, based on the introduced time-varying conversion, the stabilization and estimation with finite-time boundedness are guaranteed using the asymptotic stability
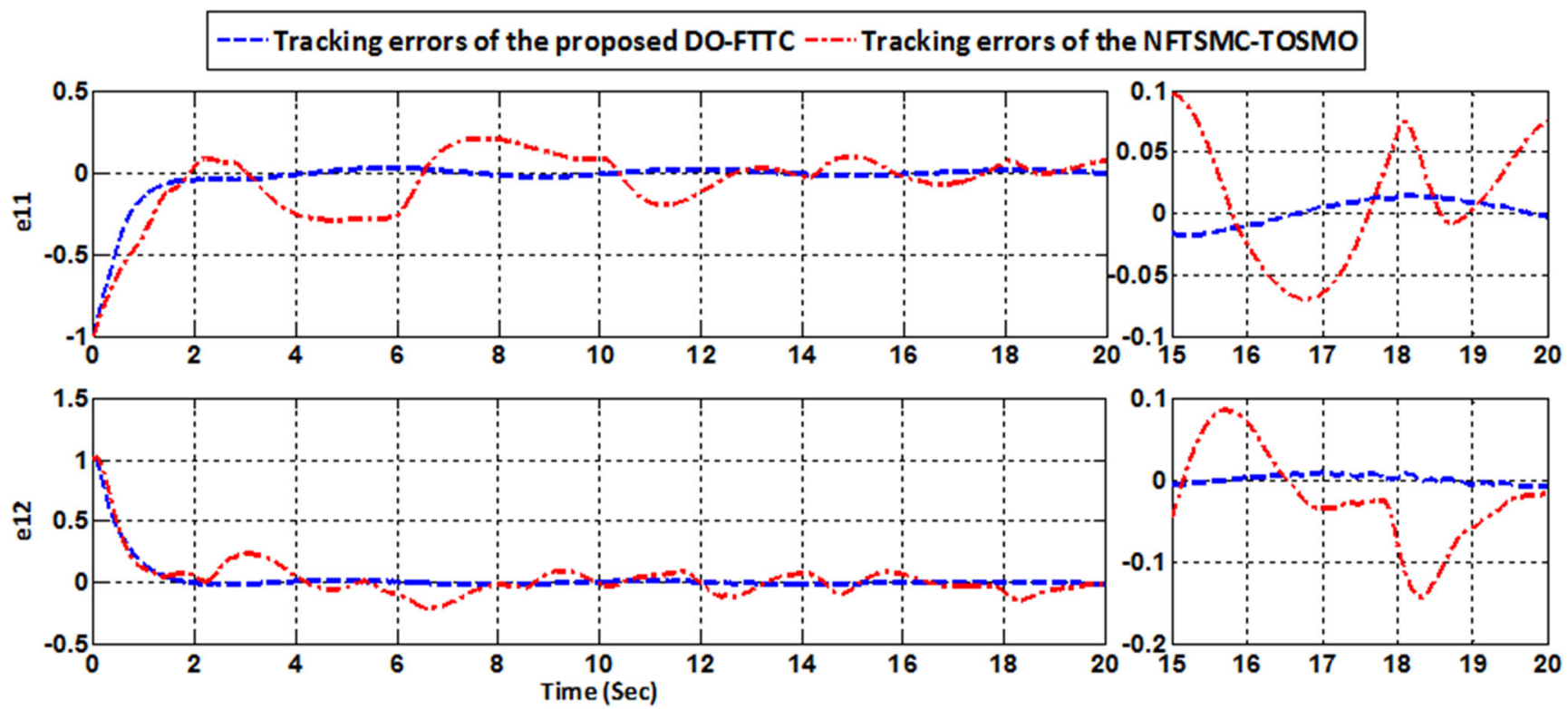

Fig. 4 Tracking error results of the closed-loop system 

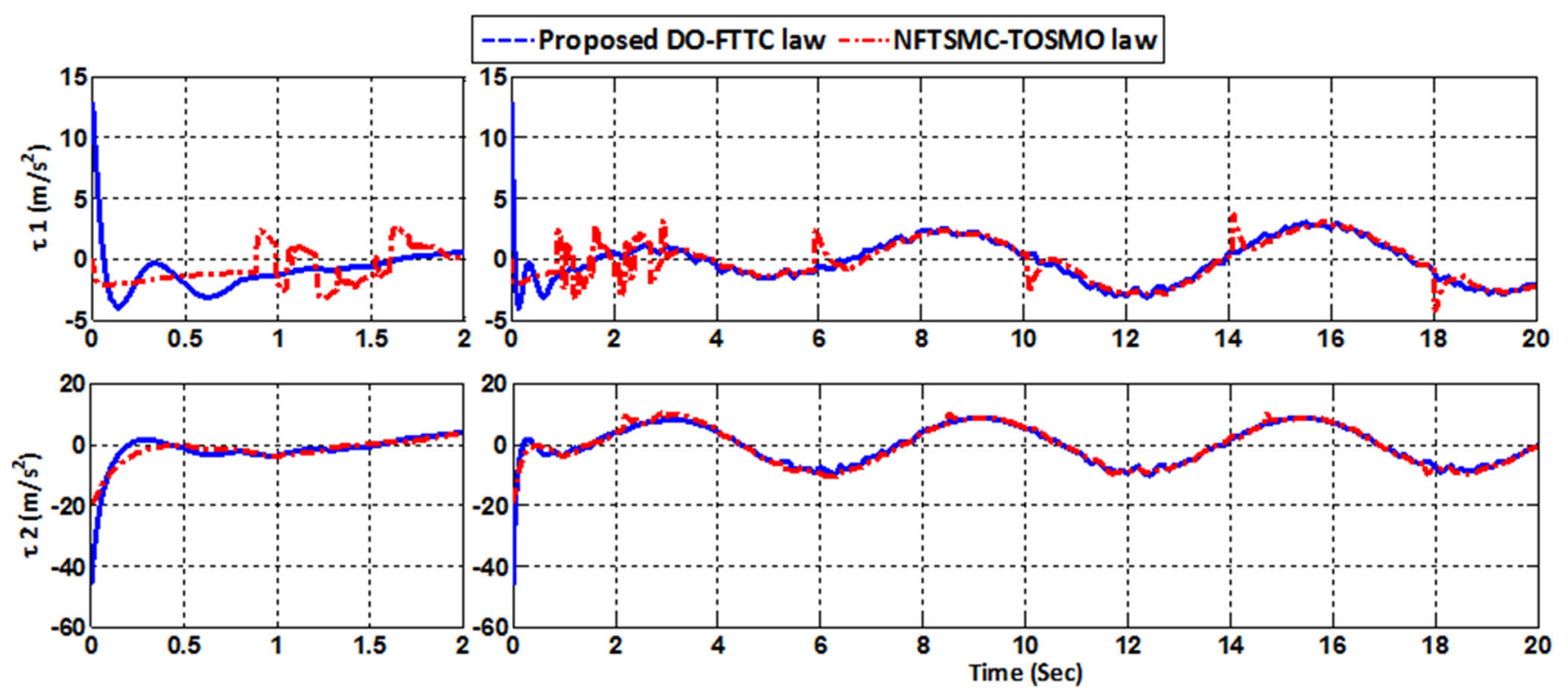

Fig. 5 Tracking control inputs

Table 1 Observers comparative results

\begin{tabular}{|c|c|c|c|}
\hline Methods & The estimation error vector $e^{\prime}$ & Perform & lance index $J_{e^{\prime}}$ \\
\hline $\begin{array}{l}\text { Proposed } \\
\text { scheme }\end{array}$ & $\begin{aligned} & e^{\prime}=(1.0 E+3) \times \\
& {\left[\begin{array}{ll}0.0000 & 0.0000 \\
0.0146 & 0.0211 \\
0.4174 & 2.0419\end{array}\right] }\end{aligned}$ & $J_{e^{\prime}}=$ & $\begin{array}{r}1.7442 E+5 \\
4.1700 E+6\end{array}$ \\
\hline $\begin{array}{c}\text { TOSMO } \\
{[24]}\end{array}$ & $\begin{array}{r}e^{\prime}=(1.0 E+3) \times \\
{\left[\begin{array}{ll}0.0071 & 0.0071 \\
0.3188 & 0.8323 \\
2.4466 & 6.5752\end{array}\right]}\end{array}$ & $J_{e^{\prime}}=$ & $\begin{array}{r}6.0878 E+6 \\
4.3926 E+7\end{array}$ \\
\hline
\end{tabular}

analyses in a straightforward manner. Finally, the time-varying gains have been computed based on straightforward algebraic equations.

\section{Conclusion}

In this paper, a time-varying chattering-free disturbance observer-based position tracking control law of serial robotic manipulators was presented to track reference signals in a finite time. As the main innovation of this paper, the robotic manipulator's model was transformed into a new time-varying form to guarantee the finite-time boundedness using the firstorder sliding mode method. Then, without any knowledge about the upper bounds of the uncertainties, a reduced-order observer was presented to estimate the uncertainties in a finite time. In this regard, time-varying gains of the observer were designed in a straightforward manner. Subsequently, a time-varying chattering-free disturbance observer-based position tracking control law was designed. The time-varying gains were provided to ensure that without any knowledge about the upper bounds of the uncertainties, the position tracking errors converge to a neighborhood of zero in a finite time and without any chattering. Finally, comparative simulations were presented to analyze the effectiveness of the proposed scheme. For future works, further development of the proposed finite-time scheme will be carried out to update the time-varying observer and controller gains based on the observation and tracking errors, respectively. In terms of more interesting topics, an optimal algorithm is suggested to be designed for choosing all the observer parameters.

Table 2 Observer-based controllers comparative results

\begin{tabular}{llll}
\hline Methods & The tracking error vector $e$ & Performance index $J_{e}$ & Performance index $J_{\tau}$ \\
\hline Proposed DO-FTTC & $e=\left[\begin{array}{l}31.7015 \\
27.7695\end{array}\right]^{T}$ & $J_{e}=1.7761 E+3$ & $J_{\tau}=1.4545 E+10$ \\
NFTSMC-TOSM [24] & $e=\left[\begin{array}{l}78.4130 \\
61.1329\end{array}\right]^{T}$ & $J_{e}=9.8858 E+3$ & $J_{\tau}=2.0465 E+10$ \\
\end{tabular}


Moreover, output feedback control can be considered to investigate the possibility of decreasing the number of physical sensors to control the robotic manipulators.

Code or Data Availability The data shown in the figures are available from the author upon request. Other datasets were not generated or analyzed during the current study.

Authors' Contributions All authors wrote and approved the manuscript. Hamid Razmjooei: Conceptualization, Methodology, Software, Validation, Formal analysis, Investigation, Resources, Data curation, Writing - original draft, Writing - review \& editing, Visualization, Project administration, Funding acquisition. Mohammad Hossein Shafiei, Gianluca Palli, and Mohammad Mehdi Arefi: Conceptualization, Methodology, Resources, Writing - review \& editing, Supervision, Project administration.

\section{Declarations}

Ethics Declarations This research work did not involve human participants or animals. Hence, ethics approval, Consent to participate and Consent for publication are not applicable.

Ethics Approval The submitted work is original and not have been published elsewhere in any form or language.

Consent to Participate Not applicable.

\section{Consent for Publication Not applicable.}

\section{Conflicts of interest/Competing interests}

The authors declare that they have no conflict of interest. Also, the authors declare that they have no known competing financial interests or personal relationships that could have appeared to influence the work reported in this paper.

Open Access This article is licensed under a Creative Commons Attribution 4.0 International License, which permits use, sharing, adaptation, distribution and reproduction in any medium or format, as long as you give appropriate credit to the original author(s) and the source, provide a link to the Creative Commons licence, and indicate if changes were made. The images or other third party material in this article are included in the article's Creative Commons licence, unless indicated otherwise in a credit line to the material. If material is not included in the article's Creative Commons licence and your intended use is not permitted by statutory regulation or exceeds the permitted use, you will need to obtain permission directly from the copyright holder. To view a copy of this licence, visit http://creativecommons.org/licenses/by/4.0/.

\section{References}

1. Tavasoli, A., Eghtesad, M., Jafarian, H.: Time-two scale control and observer design for trajectory tracking of two cooperating robot manipulators moving a flexible beam. Robot. Auton. Syst. 57(2), 212-221 (2009). https://doi.org/10.1016/j.robot.2008.04.003

2. Khalil, H., Grizzle, J.: Nonlinear Systems. Prentice hall, Upper Saddle River (2002)
3. Razmjooei, H., Shafiei, M.H.: A new approach to design a robust partial control law for the missile guidance problem. Control Eng. Appl. Inform. 18(1), 3-10 (2016)

4. Osuna-ibarra, L., Caballero-Baragan, H., Loukianov, A.G., BayroCorrochano, E.: Tracking control using optimal discrete-time H-inf for mechanical systems: applied to robotics. Robot. Auton. Syst. 119, 201-208 (2019). https://doi.org/10.1016/j.robot.2019.07.005

5. Razmjooei, H., Shafiei, M.H.: A novel finite-time disturbance observer-based partial control design: A guidance application. J. Vib. Control. 26(11-12), 1001-1011 (2020). https://doi.org/10. 1177/1077546319890951

6. Razmjooei, H., Shafiei, M.H.: Partial finite-time stabilization of perturbed nonlinear systems based on the novel concept of nonsingular terminal sliding mode method. J. Comput. Nonlinear Dyn. 15(2), 021005 (2020). https://doi.org/10.1115/1.4045632

7. Shi, D., Zhang, J., Sun, Z., Shen, G., Xia, Y.: Composite trajectory tracking control for robot manipulator with active disturbance rejection. Control. Eng. Pract. 106, 104670 (2020). https://doi.org/10. 1016/j.conengprac.2020.104670

8. Razmjooei, H., Shafiei, M.H.: A new approach to design a finite-time extended state observer: uncertain robotic manipulators application. Int. J. Robust Nonlinear Control. 31(4), 1288-1302 (2020). https://doi.org/10.1002/rnc.5346

9. Su, Y., Zheng, C., Mercorelli, P.: Robust approximate fixed-time tracking control for uncertain robot manipulators. Mech. Syst. Signal Process. 135, 106379 (2019). https://doi.org/10.1016/j. ymssp.2019.106379

10. Chen, C., Zhang, C., Hu, T., Ni, H., Chen, Q.: Finite-time tracking control for uncertain robotic manipulators using backstepping method and novel extended state observer. Int. J. Adv. Robot. Syst. 16(3), 1-15. https://doi.org/10.1177/1729881419844655

11. Tang, Y.: Terminal sliding mode control for rigid robots. Automatica. 34(1), 51-56 (1998). https://doi.org/10.1016/S00051098(97)00174-X

12. Hong, Y., Xu, Y., Huang, J.: Finite-time control for robot manipulators. Syst. Control Lett. 46(4), 243-253 (2002). https://doi.org/10. 1016/S0167-6911(02)00130-5

13. Feng, Y., Yu, X., Man, Z.: Non-singular terminal sliding mode control of rigid manipulators. Automatica. 38(12), 2159-2167 (2002). https://doi.org/10.1016/S0005-1098(02)00147-4

14. Yu, S., Yu, X., Shirinzadeh, B., Man, Z.: Continuous finite-time control for robotic manipulators with terminal sliding mode. Automatica. 41(11), 1957-1964 (2005). https://doi.org/10.1016/j. automatica.2005.07.001

15. Basin, M.V., Yu, P., Shtessel, Y.B.: Hypersonic missile adaptive sliding mode control using finite-and fixed-time observers. IEEE Trans. Ind. Electron. 65(1), 930-941 (2017). https://doi.org/10. 1109/TIE.2017.2701776

16. Jbara, A., Levant, A., Hanan, A.: Filtering homogeneous observers in control of integrator chains. Int. J. Robust Nonlinear Control. 31(9), 3658-3685 (2020). https://doi.org/10.1002/rnc.5295

17. Wang, L., Gao, Z., Zhou, X., Han, Z.: Exponential stabilization of a star-shaped thermoelastic network system based on the extended state observer with time-varying gains. IEEE Trans. Automat. Contr. 66(1), 267-274. https://doi.org/10.1109/TAC.2020. 2976317

18. Won, D., Kim, W., Tomizuka, M.: Nonlinear control with highgain extended state observer for position tracking of electro-hydraulic systems. IEEE/ASME Trans. Mechatron. 25(6), 2610-2621 (2020). https://doi.org/10.1109/TMECH.2020.2985619

19. Djennoune, S., Bettayeb, M., Al-Saggaf, U.M.: Modulating function-based fast convergent observer and output feedback control for a class of non-linear systems. IET Control Theory Appl. 13(16), 2681-2693 (2019). https://doi.org/10.1049/iet-cta.2018. 5313 
20. Razmjooei, H., Shafiei, M.H., Abdi, E.: A novel continuous finitetime extended state observer Design for a Class of uncertain nonlinear systems. IEEE Access. 8, 228289-228302 (2020). https:// doi.org/10.1109/ACCESS.2020.3043725

21. Holloway, J., Krstic, M.: Prescribed-time output feedback for linear systems in controllable canonical form. Automatica. 107, 77-85 (2019). https://doi.org/10.1016/j.automatica.2019.05.027

22. Razmjooei, H., Shafiei, M.H., Palli, G., Ibeas, A.: Chattering-free robust finite-time output feedback control scheme for a class of uncertain non-linear systems. IET Control Theory Appl. 14(19), 3168-3178 (2020). https://doi.org/10.1049/iet-cta.2020.0910

23. Razmjooei, H., Shafiei, M.H., Abdi, E., Yang, C.: A novel robust finite-time tracking control of uncertain robotic manipulators with disturbances. J. Vib. Control. (2020). https://doi.org/10.1177/ 1077546320982449

24. Nguyen, V., Vo, A., Kang, H.: A non-singular fast terminal sliding mode control based on third-order sliding mode observer for a class of second-order uncertain nonlinear systems and its application to robot manipulators. IEEE Access. 8, 78109-78120 (2020). https:// doi.org/10.1109/ACCESS.2020.2989613

25. Tylavsky, D.J., Sohie, G.R.: Generalization of the matrix inversion lemma. Proc. IEEE. 74(7), 1050-1052 (1986). https://doi.org/10. 1109/PROC.1986.13587

Publisher's Note Springer Nature remains neutral with regard to jurisdictional claims in published maps and institutional affiliations.

Hamid Razmjooei received the M.Sc. and Ph.D. degrees in Control Engineering from Shiraz University of Technology, Iran, in 2015 and 2021 , respectively. He is currently a scientific researcher in robotic technologies with the University of Bologna, Bologna, Italy. His current research interests include nonlinear, robust, and optimal control of manipulation devices, mobile manipulation, and soft robotics.

Mohammad Hossein Shafiei received the B.Sc. and M.Sc. degrees in Electronics Engineering from Shiraz University, Shiraz, Iran, in 2002 and 2005, respectively, and the Ph.D. degree in Control Engineering from the University of Tehran, Tehran, Iran, in 2010. He is currently a full Professor of the Faculty of Electrical Engineering, Shiraz University of Technology, and Shiraz, Iran. His current research interests include nonlinear control, robust and optimal methods, and switching systems.

Gianluca Palli received the Laurea and Ph.D. degrees in automation engineering from the University of Bologna, Bologna, Italy, in 2003 and 2007, respectively. He is currently a full Professor with the University of Bologna, Bologna, Italy. He has authored or coauthored more than 100 scientific articles presented at conferences or published in journals. His research interests include design and control of manipulation devices, mobile manipulation, manipulation of deformable objects, compliant actuation, and soft robotics.

Mohammad Mehdi Arefi received the B.Sc. degree from the Department of Electrical Engineering, Shiraz University, Shiraz, Iran, in 2004, and the M.Sc. and Ph.D. degrees from the Electrical Engineering Department, Iran University of Science and Technology, Tehran, Iran, in 2007 and 2011, respectively. He is an Associate Professor with the Department of Power and Control Engineering, School of Electrical and Computer Engineering, Shiraz University. His current research interests include adaptive control, robust control, nonlinear control, system identification, and application of control methods in power systems. He is in the editorial board of some journals, including the Iranian Journal of Science and Technology, Transactions of Electrical Engineering, and Industrial Control Magazine. 